\title{
Sutureless and rapid deployment implantation in bicuspid aortic valve: results from the sutureless and rapid-deployment aortic valve replacement international registry
}

\begin{abstract}
Antonio Miceli ${ }^{1}$, Paolo Berretta ${ }^{2}$, Antonio Fiore ${ }^{3}$, Martin Andreas ${ }^{4}$, Marco Solinas ${ }^{5}$, Giuseppe Santarpino ${ }^{6,7,8}$, Utz Kappert $^{9}$, Martin Misfeld ${ }^{10,11}$, Carlo Savini ${ }^{12}$, Alberto Albertini ${ }^{13}$, Emmanuel Villa ${ }^{14}$, Kevin Phan ${ }^{15}$, Theodor Fischlein $^{8}$, Bart Meuris ${ }^{16}$, Gianluca Martinelli ${ }^{17}$, Kevin Teoh ${ }^{18}$, Carmelo Mignosa ${ }^{19}$, Malakh Shrestha $^{20}$, Thierry P. Carrel ${ }^{21}$, Tristan D. Yan ${ }^{11,15}$, Mattia Glauber ${ }^{1}$, Marco Di Eusanio ${ }^{2,15}$

${ }^{1}$ Minimally Invasive Cardiac Surgery, Istituto Clinico Sant'Ambrogio, Milan, Italy; ${ }^{2}$ Cardiac Surgery Unit, Lancisi Cardiovascular Center, Polytechnic University of Marche, Ospedali Riuniti, Ancona, Italy; ${ }^{3}$ Department of Cardiac Surgery, Henri Mondor University Hospital, Assistance Publique-Hôpitaux de Paris, Créteil, France; ${ }^{4}$ Department of Cardiac Surgery, Medical University of Vienna, Vienna, Austria; ${ }^{5}$ Department of Cardiac Surgery, Pasquinucci Heart Hospital, Massa, Italy; ${ }^{6}$ Department of Cardiac Surgery, Città di Lecce Hospital, GVM Care \& Research, Lecce, Italy; ${ }^{7}$ Cardiac Surgery Unit, Department of Experimental and Clinical Medicine, Magna Graecia University, Catanzaro, Italy; ${ }^{8}$ Cardiovascular Center, Paracelsus Medical University, Nuremberg, Germany; ${ }^{9}$ Department of Cardiac Surgery, University Heart Centre Dresden, Dresden, Germany; ${ }^{10}$ University Clinic for Cardiac Surgery, Leipzig Heart Center, Leipzig, Germany; ${ }^{11}$ Department of Cardiothoracic Surgery, The Royal Prince Alfred Hospital, Sydney, Australia; ${ }^{12}$ Cardiac Surgery Department, Sant'Orsola Malpighi Hospital, University of Bologna, Bologna, Italy; ${ }^{13}$ Cardiovascular Surgery Unit, Maria Cecilia Hospital, GVM Care \& Research, Cotignola, Italy; ${ }^{14}$ Department of Cardiac Surgery, Poliambulanza Foundation Hospital, Brescia, Italy; ${ }^{15}$ The Collaborative Research (CORE) Group, Macquarie University, Sydney, Australia; ${ }^{16}$ Gasthuisberg, Cardiale Heelkunde, Leuven, Belgium; ${ }^{17}$ Cardiovascular Department, Clinica San Gaudenzio, Novara, Italy; ${ }^{18}$ Southlake Regional Health Centre, Ontario, Canada; ${ }^{19}$ Department for the Treatment and Study of Cardiothoracic Diseases and Cardiothoracic Transplantation IRCCS-ISMETT, Palermo, Italy; ${ }^{20}$ Division of Cardiothoracic Surgery, Transplantation and Vascular Surgery, Hannover Medical School, Hannover, Germany; ${ }^{21}$ Department of Cardiovascular Surgery, University Hospital, University of Bern, Bern, Switzerland

Correspondence to: Antonio Miceli, MD, PhD. Minimally invasive cardiac surgery, Istituto Clinico Sant'Ambrogio, Via Faravelli 16, Milan, Italy. Email: antoniomiceli79@alice.it.
\end{abstract}

Background: Benefits of sutureless and rapid deployment (SURD) bioprostheses in bicuspid aortic valves (BAV) are controversial. The aim of this study is to report the outcomes of patients undergoing aortic valve replacement (AVR) for BAV from the Sutureless and Rapid-Deployment Aortic Valve Replacement International Registry (SURD-IR).

Methods: Of the 4,636 patients who received primary isolated SURD-AVR between 2007 and 2018, 191 (4.1\%) BAV patients underwent AVR with SURD valve.

Results: Overall 30 -day mortality was $1.6 \%$. The Intuity valve was implanted in $53.9 \%$ of cases, whereas the Perceval was implanted in $46.1 \%$. Rate of stroke for isolated AVR was $4.2 \%$. No case of endocarditis, thromboembolism, myocardial infarction, valve dislocation or structural valve deterioration was reported in the early phase. Rate of pacemaker implantation and moderate-severe aortic regurgitation (AR) were $7.9 \%$ and $3.7 \%$, respectively.

Conclusions: BAV is not considered a contraindication for the implantation of SURD valves. However, detailed information of aortic root geometry as well as the knowledge of some technical considerations are mandatory for a good outcome.

Keywords: Bicuspid aortic valve (BAV); aortic valve replacement (AVR); sutureless; rapid deployment valve

Submitted Mar 02, 2020. Accepted for publication Jun 25, 2020.

doi: 10.21037/acs-2020-surd-33

View this article at: http://dx.doi.org/10.21037/acs-2020-surd-33 


\section{Introduction}

Bicuspid aortic valve (BAV) is a common congenital defect which occurs in $1-2 \%$ of the general population. BAV is composed of two instead of three leaflets, and often associated with one raphe in one cusp. The most common morphology is the type 1 according to the Sievers classification (1). These leaflet alterations often induce progressive fibrocalcific stenosis requiring surgery. Interestingly, it has been estimated that more than $50 \%$ of all aortic valve replacements (AVR) for aortic stenosis are related to BAV (2).

Sutureless and rapid deployment (SURD) valves represent an excellent treatment option for surgical AVR, as they simplify the surgical procedure and reduce operative times (3-7). However, in the setting of BAV, these benefits are less clear. Despite several studies demonstrating good clinical outcomes, some reports suggest that SURD valves may increase the risk of paravalvular regurgitation and/or potential dislocation related to BAV aortic root asymmetry $(8-12)$. Therefore, the aim of our study is to report the outcomes of patients undergoing AVR with SURD valves in patients with BAV from the Sutureless and RapidDeployment Aortic Valve Replacement International Registry (SURD-IR).

\section{Methods}

\section{SURD-IR}

The SURD-IR was established in 2015, enrolling patients at eighteen cardiac centres in Europe, Australia, and Canada. The study population was defined as patients undergoing SURD-AVR intervention using any available sutureless and rapid-deployment valve prosthesis either by conventional sternotomy or a less invasive approach. Valve prosthesis types included Perceval (Livanova PLC, London, UK) EDWARDS INTUITY/INTUITY Elite (Edwards Lifesciences, Irvine, CA, USA) and Enable 3F (Medtronic, MN, USA). AVR was performed via full sternotomy, ministernotomy and minithoracotomy. Details of the site selection and invitation as well as endpoints have been previously reported (5). Briefly, centers that had published reports on more than 50 SURD-AVR cases were initially invited to participate in the present database, as this was hypothesized to represent experienced centers with quality data collection. Further institutions recommended by the IVSSG Research Steering Committee were also invited to participate in the retrospective registry. Ethics approval was obtained at each of the participating centers, and datasets were submitted according to predefined spreadsheet format. Participating SURD-IR centers enrolled between 40 and 735 patients and collected information on demographics, patient comorbidities, functional status, imaging studies, surgical data, postoperative course, clinical and hemodynamic outcomes. Following electronic data submission, each dataset was evaluated to ensure that all patients were older than eighteen years. All variables between datasets were assessed with identical variables collated into a centralized database. Isolated variables reported by less than $25 \%$ of centers were excluded from analysis. Individually missing data and centre-specific nonreported data were coded separately. Clinically important absent data were queried with the submitting centre. Data were analyzed for clinical face validity and internal validity. Submitted clinical data were compared against published data for inconsistencies.

\section{Patients selection}

We examined 4,636 patients enrolled in the registry between April 2007 and December 2019. Exclusion criteria were tricuspid aortic valve, reoperative AVR and implantation of the off-market Enable 3F valve. The final sample included 191 patients with BAV who underwent AVR.

\section{Statistical analysis}

Continuous data were expressed as mean \pm standard deviation and categorical variables as percentages. The Kolmogorov-Smirnov test was used to check for normality of data. Where continuous variables did not follow a normal distribution, the median and interquartile range were reported.

\section{Results}

Of the 4,636 patients who underwent AVR between 2007 and 2019, a total of 191 patients with BAV (4.1\%) received SURD valves. Baseline characteristics are reported in Table 1. There were $92(48.2 \%)$ male patients and overall mean age was $70.7 \pm 9.8$ with a median EuroSCORE I and II of $5.8 \%(3.3-8.7 \%)$ and $1.6 \%(1-2.6 \%)$, respectively. The indication for AVR was aortic stenosis in $56 \%$ of the patients, mixed disease in $43.5 \%$ and regurgitation in $0.5 \%$. Mean LVEF was $57.8 \% \pm 9.9 \%$ and 3 patients presented with LVEF $<30 \%$. 


\begin{tabular}{|c|c|}
\hline Characteristics & Data \\
\hline Male & $92(48.2)$ \\
\hline Age & $70.7 \pm 8.1$ \\
\hline \multicolumn{2}{|l|}{ NYHA class } \\
\hline 1 & $14(7.4)$ \\
\hline II & $68(36.2)$ \\
\hline III & $98(52.1)$ \\
\hline IV & $8(4.3)$ \\
\hline Hypertension & $105(67.3)$ \\
\hline Obesity & $39(20.5)$ \\
\hline BMI & $26.6 \pm 4.5$ \\
\hline Diabetes & $37(22.0)$ \\
\hline Dyslipidemia & $75(49.0)$ \\
\hline AF & $18(13.7)$ \\
\hline LVEF\% & $57.8 \pm 9.9$ \\
\hline LVEF > $>50$ & $157(83.1)$ \\
\hline LVEF 30-50 & $29(15.3)$ \\
\hline LVEF $<30$ & $3(1.6)$ \\
\hline Aortic valve regurgitation & $1(0.5)$ \\
\hline Mixed aortic valve disease & $83(43.5)$ \\
\hline Cerebrovascular disease & $15(8.3)$ \\
\hline Renal insufficiency & $50(27.0)$ \\
\hline Dialysis & $1(0.6)$ \\
\hline CAD & $44(28.0)$ \\
\hline Chronic lung disease & $37(20.1)$ \\
\hline Redo & $6(3.1)$ \\
\hline Urgent/emergent status & $1(0.6)$ \\
\hline Logistic Euroscore, median (IQR) & $5.8(3.3-8.7)$ \\
\hline Euroscore II, median (IQR) & $1.6(1-2.6)$ \\
\hline \multicolumn{2}{|c|}{$\begin{array}{l}\text { Data present as } n(\%) \text {, mean } \pm \mathrm{SD} \text {, and median (IQR). NYHA, } \\
\text { New York Heart Association; BMI, body mass index; LVEF, left } \\
\text { ventricular ejection fraction; CAD, coronary artery disease; IQR, } \\
\text { interquartile range. }\end{array}$} \\
\hline
\end{tabular}

\section{Surgical procedure}

Operative data are reported in Table 2. The majority of patients underwent a minimally invasive approach (73.8\%).

\begin{tabular}{|ll}
\hline Table 2 Operative data & \\
\hline Variables & Data \\
\hline Full sternotomy & $50(26.2)$ \\
\hline Ministernotomy & $91(47.6)$ \\
\hline Right anterior minithoracotomy & $50(26.2)$ \\
\hline Conversion to full sternotomy & $2(1.4)$ \\
\hline Perceval & $88(46.1)$ \\
\hline Intuity/Intuity Elite & $103(53.9)$ \\
\hline Valve malpositioning & $1(0.5)$ \\
\hline Associate procedures & $61(31.9)$ \\
\hline CABG & $26(13.6)$ \\
\hline Mitral valve surgery & $4(2.1)$ \\
\hline Tricuspid valve surgery & $3(1.6)$ \\
\hline Maze & $6(3.1)$ \\
\hline Thoracic aorta surgery & $25(13.5)$ \\
\hline Septal myectomy & $4(2.1)$ \\
\hline Overall CPB time & 87 [65-116] \\
\hline Overall clamp time & $60[43-82]$ \\
\hline Isolated AVR (min) & 107 [79-141.5] \\
\hline CPB time & 55 [35.5-72] \\
\hline Clamp time & \\
\hline Combined & \\
\hline Clamp time & replacement; IQR, \\
\hline
\end{tabular}

Specifically, ministernotomy was performed in $47.6 \%$ of procedures, followed by right anterior minithoracotomy (26.2\%) and full sternotomy (26.2\%). Associated procedures included coronary artery bypass grafting (CABG) (13.6\%), thoracic aortic surgery $(13.5 \%)$, mitral valve surgery $(2.1 \%)$ and tricuspid annuloplasty (1.6\%). Median aortic crossclamp time was 60 [43-82] min and cardiopulmonary bypass time was 87 [65-116] $\mathrm{min}, 116.6 \pm 43.7 \mathrm{~min}$ (Table 2).

\section{Early outcomes}

Overall 30 -day mortality was $1.6 \%$. Overall rate of stroke 


\begin{tabular}{|ll}
\hline Table 3 In-hospital outcomes & \\
\hline Variables & Data \\
\hline 30-day mortality & $3(1.6)$ \\
\hline Stroke & $8(4.2)$ \\
\hline Low cardiac output & - \\
\hline Ventilatory support $>72 \mathrm{~h}$ & $4(2.1)$ \\
\hline New onset atrial fibrillation & $53(31.9)$ \\
\hline New AV block requiring PM & $15(7.9)$ \\
\hline Aortic regurgitation & $19(9.9)$ \\
\hline Mild & $12(6.1)$ \\
\hline Moderate & $6(3.3)$ \\
\hline Severe & $1(0.6)$ \\
\hline Bleeding requiring revision & $6(3.1)$ \\
\hline AKI > stage 1 & $6(3.1)$ \\
\hline Dialysis & - \\
\hline Wound complications & $7(4.5)$ \\
\hline ICU stay (days) & 12 [9-16] \\
\hline Hospital stay (days) & \\
\hline Data present as $n$ (\%) and median [IQR]. AV, atrio ventricular; \\
\hline range. & IQR, interquartile \\
\hline
\end{tabular}

was $4.2 \%$ (isolated AVR $3.8 \%$ and combined procedure $4.9 \%$ ). Rate of $\mathrm{AV}$ block requiring pacemaker and atrial fibrillation were $7.9 \%$ and $31.9 \%$, respectively. No cases of endocarditis, thromboembolism, myocardial infarction or structural valve deterioration were reported in the early phase. Median length of stay in the intensive care unit and hospital stay were 2 (IQR 1-4) and 12 [9-16] days, respectively. Other outcomes are reported in Table 3.

\section{Valve implanted}

The Intuity valve was implanted in $53.9 \%$ of cases, whereas the Perceval was implanted in $46.1 \%$. One case of Perceval valve malpositioning was reported, which required a second cross clamp and the same Perceval was repositioned. The most common size implanted for Perceval was large (36.3\%), followed by medium (29.5\%), extra large (26.1\%) and small (7.9\%). For Intuity, the most common size implanted was 23 (32\%), followed by 25 (25.2\%), 27 (17.5\%), 21 (13.6\%) and $19(11.6 \%)$ (Table 4). Overall rate of pacemaker

\begin{tabular}{lll} 
Table 4 Valve prostheses: sizes and hemodynamics $(\mathrm{n}=191)$ \\
\hline & $\begin{array}{l}\text { Peak gradient } \\
(\mathrm{mmHg}), \\
(\mathrm{mean} \pm \mathrm{SD})\end{array}$ & $\begin{array}{l}\text { Mean gradient } \\
(\mathrm{mmHg}) \\
(\mathrm{mean} \pm \mathrm{SD})\end{array}$ \\
\hline Perceval & $28.3 \pm 10.9$ & $14.8 \pm 5.8$ \\
Small & $35.4 \pm 8.8$ & $18.5 \pm 3.9$ \\
Medium & $32.6 \pm 9.2$ & $16.7 \pm 4.9$ \\
Large & $26.8 \pm 12.9$ & $14.4 \pm 6.9$ \\
Extra-large & $22.6 \pm 6.1$ & $11.5 \pm 3.6$ \\
EDWARDS INTUITY/ & $19.2 \pm 7$ & $10.9 \pm 4.4$ \\
INTUITY Elite & & $14.1 \pm 5.2$ \\
$19 \mathrm{~mm}$ & $23.9 \pm 8.4$ & $12.3 \pm 6.4$ \\
$21 \mathrm{~mm}$ & $20.6 \pm 10.8$ & $10.4 \pm 3.4$ \\
$23 \mathrm{~mm}$ & $18.4 \pm 5.5$ & $9.5 \pm 3.1$ \\
$25 \mathrm{~mm}$ & $18.4 \pm 5.5$ & $9.7 \pm 3.4$ \\
$27 \mathrm{~mm}$ & $17.1 \pm 4.8$ & \\
\hline
\end{tabular}

implantation was $7.9 \%$ and was slightly higher in the Intuity group $(9.7 \%$ vs. $5.7 \%, \mathrm{P}=0.446)$. Overall rate of aortic regurgitation (AR) was $9.9 \%$ and similar between groups (Table 5). Rate of moderate and severe AR was 3.7\%

\section{Discussion}

Our study demonstrated that in patients with BAV disease, AVR with either sutureless or rapid deployment valves is a safe procedure, associated with excellent outcomes and hemodynamic performances. Specifically, overall 30-day mortality was $1.6 \%$, which reflected the predicted median Euroscore II. Furthermore, no cases of endocarditis, thromboembolism, myocardial infarction or structural valve deterioration were reported in the early phase. Interestingly, the majority of these procedures were performed through a minimally invasive approach, reinforcing the concept that SURD valves are not contraindicated in BAV. We reported only one case of valve migration (Perceval) which required a second cross clamp for repositioning the same valve. This is the largest case series study reporting outcomes of patients with BAV undergoing AVR with the Perceval or Intuity valve. These valves have been developed as an alternative to conventional, stented valves to reduce operative times and standardize the surgical procedure (3-7). Outcomes are excellent up to 10 years; however, these results focused 


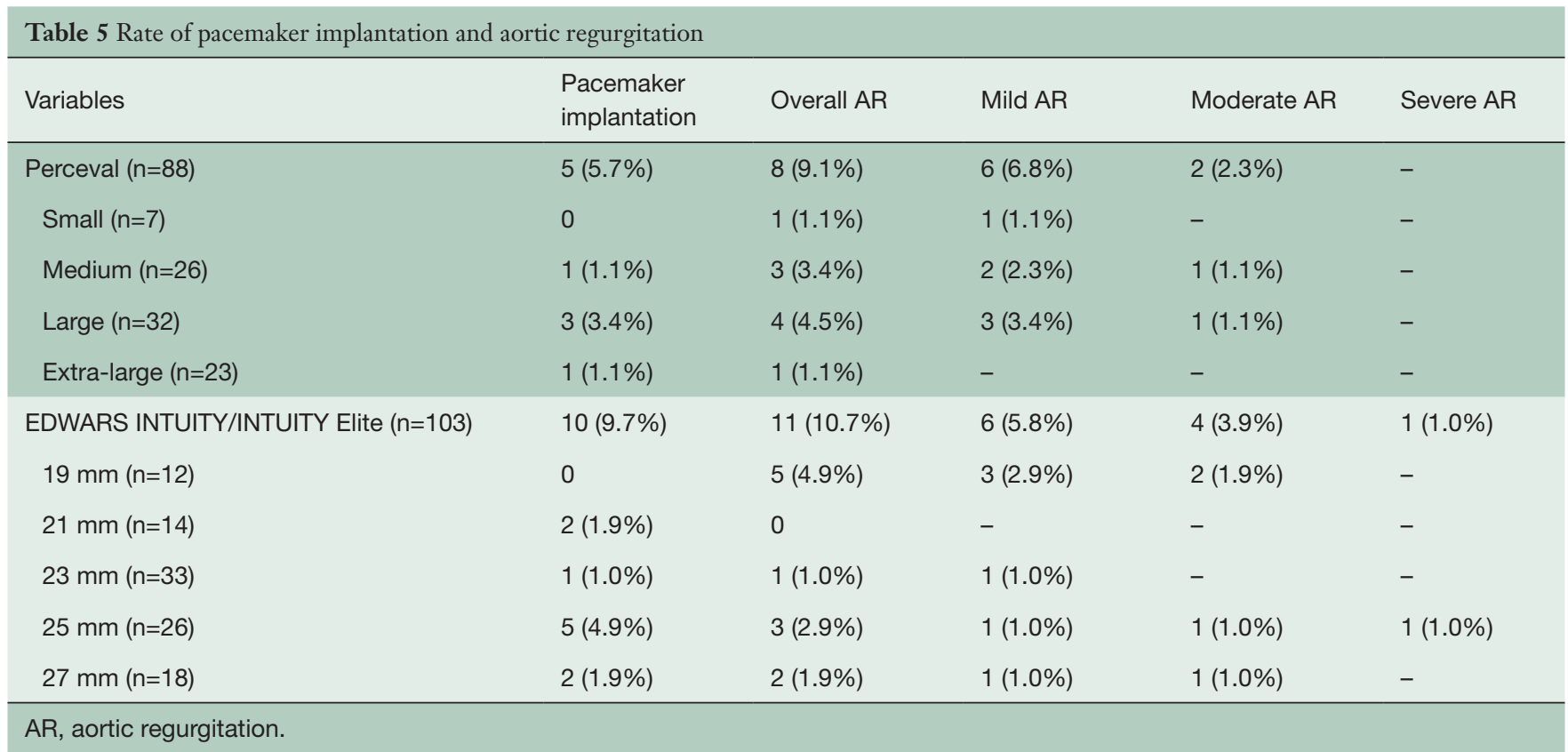

mainly on patients presenting with tricuspid aortic valve $(3,6,13)$. BAV has been considered a contraindication for the implantation of the SURD valve, as it is often associated with altered geometric root, sinus asymmetry, elliptic annulus and different commissural heights. In addition, some cases of migration and dislocation have been reported at follow-up, suggesting some words of caution in using these prostheses in $\operatorname{BAV}(11,12,14)$. An international expert consensus panel recommends the use of CT scan in defining the anatomical root geometry for evaluating aortic annulus, sinotubular junction and leaflet morphology (15). In the presence of $\mathrm{BAV}$, experts recommend the use of SURD valves in type 1 Sievers classification as well as in some cases of type 2 when the two commissures have approximately the same height. Conversely, the absence of raphe (type 0) is considered an absolute contraindication, because of its anatomy related to asymmetric height of commissures, unequal width of the sinuses of Valsalva and $180^{\circ}$ orientation of the coronary ostia.

Nguyen et al. reported the first case series of 25 type I BAV patients and aortic stenosis receiving the Perceval valve (8). Interestingly, neither migration nor structural damage occurred at follow-up and only 3 patients had trace of AR. The key points for a perfect sealing of SURD valves are the symmetry of the aortic annulus and the equality of the heights of the leaflet commissures. Nguyen et al. suggested recreating the 3 nadir points positioned at
120 degrees as it is in a tricuspid valve. However, this technique is suitable only in type I BAV when 3 equal commissures and sinus of Valsalva are present. In all other cases, a commissural plication is required to restore the circularity of the aortic annulus, especially when commissures are placed at different heights (10). Based on this technical consideration, Durdu et al. reported 13 successful cases of AVR through minithoracotomy with Perceval in BAV, of whom 4 had type 0 and 2 type 2 BAV (9). In addition to the intercommissural triangle plication for annular remodeling, authors recommended a thorough decalcification of the aortic annulus as it restores its elasticity and adaptability to the implanted prosthesis. We finally add that the correct sizing is essential in avoiding postoperative $\mathrm{AR}$ and pacemaker implantation. The choice of a smaller valve may be associated with paravalvular leakage for the Intuity valve and central AR for the Perceval. Conversely, a larger valve might be associated with increased pacemaker implantation rate for both valves and potential hemodynamic turbulence in the case of the Perceval valve.

Rate of postoperative stroke was higher compared to other studies involving SURD and that deserves some potential explanation. BAV is often heavily calcified and its debridement to restore the annular circularity may have contributed this result. Furthermore, one patient had a previous stroke. Finally, this rate was higher in combined procedures ( $3.8 \%$ vs. $4.9 \%)$. It is well known 
that combined procedures are associated with a higher complication risk $(16,17)$.

The rate of significant postoperative AR was $3.7 \%$. This data is in the line with others. However, when compared with the overall population of SURD registry, we found 2.5 times the increased risk of postoperative AR, highlighting the complexity of BAV anatomy and the importance of restoring the annular circumference for a perfect result (5).

The incidence of pacemaker (PMK) implantation was $7.9 \%$ (15 patients). Several mechanisms may be associated with PMK implantation, the most common contributing factor being mechanical trauma related to decalcification and the expansion of both valves at the level of left ventricular outflow tract. However, extensive debridement of the aortic annulus and center timing policy for PMK implantation may have also contributed (18). Finally, the absolute number of pacemaker implantations was low, therefore we were not able to evaluate a potential improvement of this rate over the years.

This study presents several limitations. First, it is based on the retrospective analysis of the SURD international registry. Therefore, we were unable to account for influence of any residual unmeasured factors that could affect the adverse outcomes. Second, our database does not account for the Sievers classification and it is not possible to investigate between BAV type and AR. Then, our database was not able to distinguish paravalvular leak or central regurgitation. Finally, no follow-up is available and therefore we do not know about any potential cases of dislocation/migration.

In conclusion, BAV itself is not a contraindication for implantation of SURD valve. However, a word of caution is required. Even though we dealt with any type of BAV, AVR with SURD in type 0 BAV is technically demanding. Detailed information of aortic root geometry as well as the knowledge of some technical considerationsdecalcification, sizing, and annular remodeling-are mandatory for a good outcome.

\section{Acknowledgments}

None.

\section{Footnote}

Conflicts of Interest: Antonio Miceli: Livanova consultant; Martin Andreas is a proctor for Edwards Lifesciences and Advisory Board Member for Medtronic, Inc.; Gianluca
Martinelli: proctoring and speakers services for Edwards; Carmelo Mignosa is a consultant/proctor for LivaNova; Alberto Albertini receives consulting and lecture fees from LivaNova; Marco Solinas: Livanova consultant; Mattia Glauber: Livanova consultant; Theodor Fischlein: LivaNova and BioStable consultant; Bart Mauris: Livanova and Edwards Consultant. The other authors have no conflicts of interest to declare.

Ethical Statement: Ethics approval was obtained at each of the participating centers, and datasets were submitted according to predefined spreadsheet format.

Open Access Statement: This is an Open Access article distributed in accordance with the Creative Commons Attribution-NonCommercial-NoDerivs 4.0 International License (CC BY-NC-ND 4.0), which permits the noncommercial replication and distribution of the article with the strict proviso that no changes or edits are made and the original work is properly cited (including links to both the formal publication through the relevant DOI and the license). See: https://creativecommons.org/licenses/by-nc-nd/4.0/.

\section{References}

1. Sievers HH, Schmidtke C. A classification system for the bicuspid aortic valve from 304 surgical specimens. J Thorac Cardiovasc Surg 2007;133:1226-33.

2. Michelena HI, Prakash SK, Della Corte A, et al. Bicuspid aortic valve: identifying knowledge gaps and rising to the challenge from the International Bicuspid Aortic Valve Consortium (BAVCon). Circulation 2014;129:2691-704.

3. Shrestha M, Fischlein T, Meuris B, et al. European multicentre experience with the sutureless Perceval valve: clinical and haemodynamic outcomes up to 5 years in over 700 patients. Eur J Cardiothorac Surg 2016;49:234-41.

4. Powell R, Pelletier MP, Chu MWA, et al. The Perceval Sutureless Aortic Valve: Review of Outcomes, Complications, and Future Direction. Innovations (Phila) 2017;12:155-73.

5. Di Eusanio M, Phan K, Berretta P, et al. Sutureless and Rapid-Deployment Aortic Valve Replacement International Registry (SURD-IR): early results from 3343 patients. Eur J Cardiothorac Surg 2018;54:768-73.

6. Laufer G, Haverich A, Andreas M, et al. Long-term outcomes of a rapid deployment aortic valve: data up to 5 years. Eur J Cardiothorac Surg 2017;52:281-7.

7. Phan K, Tsai YC, Niranjan N, et al. Sutureless aortic valve 
replacement: a systematic review and meta-analysis. Ann Cardiothorac Surg 2015;4:100-11.

8. Nguyen A, Fortin W, Mazine A, et al. Feasibility and outcomes of sutureless valve replacement in patients with bicuspid aortic valves. J Thorac Cardiovasc Surg 2015;150:851-7.

9. Durdu MS, Gumus F, Ozcinar E, et al. Sutureless Valve Replacement Through a Right Anterior Mini-thoracotomy in Elderly Patients With Stenotic Bicuspid Aortic Valve. Semin Thorac Cardiovasc Surg 2019;31:458-64.

10. Glauber M, Ferrarini M, Lio A, et al. Dealing with a stenotic bicuspid aortic valve: Is this still an off-label procedure for a sutureless valve? J Thorac Cardiovasc Surg 2015;150:858-9.

11. Chiariello GA, Villa E, Messina A, et al. Dislocation of a sutureless prosthesis after type I bicuspid aortic valve replacement. J Thorac Cardiovasc Surg 2018;156:e87-9.

12. Amir A, Moore A, Carvalho G, et al. A story of migration of a surgically implanted aortic sutureless. J Cardiothorac Vasc Anesth 2018;32:464-6.

13. Meuris B, Szecel D, Eurlings R, et al. Sutureless AVR

Cite this article as: Miceli A, Berretta P, Fiore A, Andreas M, Solinas M, Santarpino G, Kappert U, Misfeld M, Savini C, Albertini A, Villa E, Phan K, Fischlein T, Meuris B, Martinelli G, Teoh K, Mignosa C, Shrestha M, Carrel TP, Yan TD, Glauber M, Di Eusanio M. Sutureless and rapid deployment implantation in bicuspid aortic valve: results from the sutureless and rapid-deployment aortic valve replacement international registry. Ann Cardiothorac Surg 2020;9(4):298-304. doi: 10.21037/acs-2020-surd-33 experience in a single center: 11 years of use in 468 patients. Presented at 99th AATS meeting 2019 Toronto, Canada.

14. Carrier M. Replacement of bicuspid aortic valve with sutureless bioprosthesis: a word of caution here. J Thorac Cardiovasc Surg 2018;156:e91.

15. Gersak B, Fischlein T, Folliguet TA, et al. Sutureless, rapid deployment valves and stented bioprosthesis in aortic valve replacement: recommendations of an International Expert Consensus Panel. Eur J Cardiothorac Surg 2016;49:709-18.

16. Messé SR, Acker MA, Kasner SE, et al. Stroke after aortic valve surgery: results from a prospective cohort. Circulation 2014;129:2253-61.

17. Online STS Adult Cardiac Surgery Risk Calculator. Accessed on 26 February 2020. Available online: http:// riskcalc.sts.org/stswebriskcalc/calculate

18. Miceli A, Lio A, Glauber M. Size, position, and timing: A mixture of success. J Thorac Cardiovasc Surg 2016;152:633-4. 